\title{
Dual spacetime and nonsingular string cosmology
}

\author{
Robert Brandenberger, ${ }^{1, *}$ Renato Costa, ${ }^{2, \dagger}$ Guilherme Franzmann, ${ }^{1, \hbar}$ and Amanda Weltman ${ }^{2, \S}$ \\ ${ }^{1}$ Physics Department, McGill University, Montreal, Québec, H3A 2T8, Canada \\ ${ }^{2}$ Cosmology and Gravity Group, Department of Mathematics and Applied Mathematics, \\ University of Cape Town, Rondebosch 7700, South Africa
}

(Received 8 July 2018; published 18 September 2018)

\begin{abstract}
Making use of the T-duality symmetry of superstring theory and of the double geometry from double field theory, we argue that cosmological singularities of a homogeneous and isotropic universe disappear. In fact, an apparent big bang singularity in Einstein gravity corresponds to a universe expanding to infinite size in the dual dimensions.

DOI: 10.1103/PhysRevD.98.063521
\end{abstract}

\section{INTRODUCTION}

The singularities which arise at the beginning of time in both standard and inflationary cosmology indicate that the theories which are being used in cosmology break down as the singularity is approached. If space-time is described by Einstein gravity and matter obeys energy conditions which are natural from the point of view of point particle theories, then singularities in homogeneous and isotropic cosmology are unavoidable [1]. These theorems in fact extend to inflationary cosmology [2-4].

But we know that Einstein gravity coupled to point particle matter cannot be the correct description of nature. The quantum structure of matter is not consistent with a classical description of space-time. The early Universe needs to be described by a theory which can unify spacetime and matter at a quantum level. Superstring theory (see e.g., $[5,6]$ for a detailed overview) is a promising candidate for a quantum theory of all four forces of nature. At least at the string perturbative level, the building blocks of string theory are fundamental strings. Strings have degrees of freedom and new symmetries which point particle theories do not have, and these features may lead to a radically different picture of the very early Universe, as discussed many years ago in [7] (see also [8]).

As discussed in [7], string thermodynamic considerations indicate that the cosmological evolution in the context of string theory should be nonsingular. A key realization is

\footnotetext{
rhb@physics.mcgill.ca

Tenato.Santos@uct.ac.za

guilherme.franzmann@mail.mcgill.ca

sawelti@gmail.com
}

Published by the American Physical Society under the terms of the Creative Commons Attribution 4.0 International license. Further distribution of this work must maintain attribution to the author(s) and the published article's title, journal citation, and DOI. Funded by SCOAP. that the temperature of a gas of closed strings in thermal equilibrium cannot exceed a limiting value, the Hagedorn temperature [9]. In fact, as reviewed in the following section, the temperature of a gas of closed strings in a box of radius $R$ decreases as $R$ becomes much smaller than the string length. If the entropy of the string gas is large, then the range of values of $R$ for which the temperature is close to the Hagedorn temperature $T_{H}$ is large. This is called the "Hagedorn phase" of string cosmology. The exit from the Hagedorn phase is smooth and is a consequence of the decay of string winding modes into string loops. ${ }^{1}$ The transition leads directly to the radiation phase of standard big bang cosmology (see [11] for reviews of the string gas cosmology scenario).

If strings in the Hagedorn phase are in thermal equilibrium, then the thermal fluctuations of the energymomentum tensor can be computed using the methods of [12]. In particular, it can be shown that in a compact space with stable winding modes the specific heat capacity has holographic scaling as a function of the radius of the volume being considered. As a consequence [13,14], thermal fluctuations of strings in the Hagedorn phase lead to a scale-invariant spectrum of cosmological perturbations at late times, with a slight red tilt like what is predicted [15] in inflationary cosmology. If the string scale is comparable to the scale of particle physics grand unification, the predicted amplitude of the fluctuations matches the observations well (see [16] for recent observational results). Hence, string gas cosmology provides an alternative to cosmological inflation as a theory for the origin of structure in the Universe. The predicted spectrum of gravitational waves [17] is also scale invariant, but a slight blue tilt is predicted, in contrast to the

\footnotetext{
${ }^{1}$ This mechanism suggests that exactly three spatial dimensions can become large [7], the others being confined to the string length by the interaction of the string winding and momentum modes [10].
} 
prediction in standard inflationary cosmology. This is a prediction by means of which the scenario can be distinguished from standard inflation (meaning inflation in Einstein gravity driven by a matter field obeying the usual energy conditions). A simple modeling of the transition between the Hagedorn phase and the radiation phase leads to a running of the spectrum which is parametrically larger than what is obtained in simple inflationary models [18].

In this paper, we will study the cosmological background dynamics which follow from string theory if the target space has stable winding modes. An example where this is the case is a spatial torus. We will argue that from the point of view of string theory the dynamics is nonsingular.

\section{DUAL SPACE FROM T-DUALITY}

For simplicity let us assume that space is toroidal with $d=9$ spatial dimensions, all of radius $R$. Closed strings then have momentum modes whose energies are quantized in units of $1 / R$,

$$
E_{n}=\frac{n}{R},
$$

where $n$ is an integer. They also have winding modes whose energies are quantized in units of $R$, i.e.,

$$
E_{m}=m R
$$

where $m$ is an integer and we are working in units where the string length is 1 . Strings also have a tower of oscillatory modes whose energies are independent of $R$. The number of oscillatory modes increases exponentially with energy.

It follows from (1) and (2) that the spectrum of string states is invariant under the T-duality transformation

$$
R \rightarrow \frac{1}{R}
$$

if the momentum and winding numbers are interchanged. The transformation (3) is also a symmetry of the string interactions and is assumed to be a symmetry of string theory beyond perturbation theory (see, e.g., [6]). ${ }^{2}$

As is well known, the position eigenstates $|x\rangle$ are dual to momentum eigenstates $|p\rangle$. In a compact space, the momenta are discrete, labeled by integers $n$, and hence

$$
|x\rangle=\sum_{n} e^{i n x}|n\rangle,
$$

where $|n\rangle$ is the momentum eigenstate with momentum quantum number $n$. As already discussed in [7], in our string theory setting, windings are T-dual to momenta, and we can define a T-dual position operator

\footnotetext{
${ }^{2}$ See also [19] for an extended discussion of T-duality when branes are added.
}

$$
|\tilde{x}\rangle=\sum_{m} e^{i m \tilde{x}}|m\rangle
$$

where $|m\rangle$ are the eigenstates of winding, labeled by an integer $m$.

As again argued in [7], experimentalists will measure physical length in terms of the position operators which are the lightest. Thus, for $R>1$ (in string units), it is the regular position operators $|x\rangle$ which determine physical length, whereas for $R<1$ it is the dual variables $|\tilde{x}\rangle$. Hence, the physical length $l_{p}(R)$ is given by

$$
\begin{array}{ll}
l(R)=R & \text { for } R \gg 1, \\
l(R)=\frac{1}{R} & \text { for } R \ll 1 .
\end{array}
$$

As was argued in [7], in string gas cosmology the temperature singularity of the big bang is automatically resolved. If we imagine the radius $R(t)$ decreasing from some initially very large value (large compared to the string length), and matter is taken to be a gas of superstrings, then the temperature $T$ will initially increase, since for large values of $R$ most of the energy of the system is in the light modes, which are the momentum modes, and the energy of these modes increases as $R$ decreases. Before $T$ reaches the maximal temperature $T_{H}$, the increase in $T$ levels off since the energy can now go into producing oscillatory modes. For $R<1$ (in string units) the energy will flow into the winding modes which are now the light modes. Hence,

$$
T(R)=T\left(\frac{1}{R}\right)
$$

A sketch of the temperature evolution as a function of $R$ is shown in Fig. 1. As a function of $\ln R$, the curve is symmetric as a reflection of the symmetry (7). The region of $R$ when the temperature is close to $T_{H}$ and the curve in

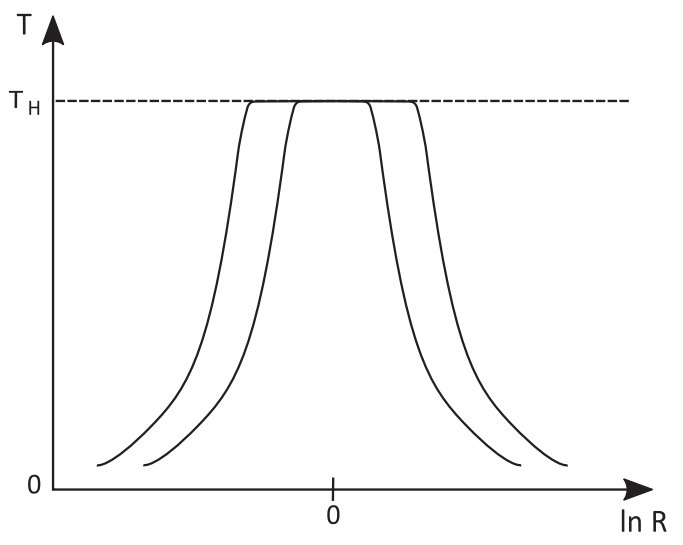

FIG. 1. T versus $\log R$ for type II superstrings. Different curves are obtained for different entropy values, which are fixed. The larger the entropy is, the larger the plateau, given by the Hagedorn temperature. For $R=1$ we have the self-dual point. 
Fig. 1 is approximately horizontal is called the Hagedorn phase. Its extent is determined by the total entropy of the system [7].

\section{COSMOLOGICAL DYNAMICS AND DUAL SPACE-TIME}

In the following we will couple a gas of strings to a background appropriate to string theory. Since the massless modes of string theory include, in addition to the graviton, the dilaton and an antisymmetric tensor field, a cosmological background will contain the metric, the dilaton, and the antisymmetric tensor field. For a homogeneous and isotropic cosmology, the metric can be written as

$$
d s^{2}=-d t^{2}+a(t)^{2} d \mathbf{x}^{2},
$$

where $t$ is physical time, $a(t)$ is the cosmological scale factor, and $\mathbf{x}$ are comoving spatial coordinates. We have assumed vanishing spatial curvature for simplicity. We denote the dilaton by $\phi(t)$.

The T-duality symmetry of string theory leads to an important symmetry of the massless background fields, the scale factor duality [20]. In the absence of an antisymmetric tensor field, these take the form

$$
\begin{aligned}
a(t) & \rightarrow \frac{1}{a} \\
\bar{\phi}(t) & \rightarrow \bar{\phi}(t),
\end{aligned}
$$

where the T-duality invariant combination of the scale factor and the dilaton is

$$
\bar{\phi} \equiv \phi-d \ln a,
$$

where $d=D-1$ is the number of spatial dimensions and $D$ the number of space-time dimensions.

The background equations of motion are those of dilaton gravity (we will neglect the antisymmetric tensor field). In the absence of matter, these equations were studied in detail in the context of pre-big-bang cosmology [20]. In the presence of string matter, they have been analyzed in [21]. The equations in the presence of a gas of matter described by energy density $\rho$ and pressure $p$ are

$$
\begin{gathered}
(\dot{\phi}-d H)^{2}-d H^{2}=e^{\phi} \rho \\
\dot{H}-H(\dot{\phi}-d H)=\frac{1}{2} e^{\phi} p \\
2(\ddot{\phi}-d \dot{H})-(\dot{\phi}-d H)^{2}-d H^{2}=0,
\end{gathered}
$$

where $H \equiv \dot{a} / a$. These are the equations in the string frame. In particular, we can combine these equations to write a continuity equation,

$$
\dot{\rho}+(D-1) H(\rho+p)=0 .
$$

We consider matter to be a gas of strings. For $R \gg 1$ most of the energy is in the momentum modes, which act as radiation and hence have an equation of state parameter $w \equiv p / \rho$ given by $w=1 / d$. For $R \ll 1$, however, most of the energy density is in the winding modes, whose equation of state parameter is $w=-1 / d$. Finally, for $R=1$ the equation of state is $w=0$. An interpolating form of the matter equation of state is

$$
w(a)=\frac{2}{\pi d} \arctan \left(\beta \ln \left(\frac{a}{a_{0}}\right)\right),
$$

where $a_{0}$ is the value of the scale factor when $R=1$, and $\beta$ is a constant which depends on the total entropy of the gas. The larger the entropy is, the wider the Hagedorn phase as a function of $a$, and hence the smaller the value of $\beta$. For this equation of state, the continuity equation for string gas matter can be integrated and yields

$$
\begin{aligned}
\ln \frac{\rho}{\rho_{0}}= & -d \ln \frac{a}{a_{0}}-\frac{2}{\pi}\left\{\ln \left(\frac{a}{a_{0}}\right) \arctan \left[\beta \ln \left(\frac{a}{a_{0}}\right)\right]\right\} \\
& -\frac{2}{\pi}\left\{\frac{1}{2 \beta} \ln \left[1+\beta^{2}\left(\ln \frac{a}{a_{0}}\right)^{2}\right]\right\},
\end{aligned}
$$

where $\rho_{0}$ is the energy density at the string length. This result reproduces what is expected for large and small radii,

$$
\begin{gathered}
\rho(a \text { large }) \rightarrow \rho_{0}\left(a / a_{0}\right)^{-(d+1)} \\
\rho(a \text { small }) \rightarrow \rho_{0}\left(a / a_{0}\right)^{-(d-1),}
\end{gathered}
$$

for pure momentum or pure winding modes, respectively.

At this point we have a system of background and matter in which both components have the same symmetries. We now turn to an exploration of solutions. Following closely [20], we make the ansatz

$$
\begin{aligned}
& a(t) \sim\left(\frac{t}{t_{0}}\right)^{\alpha} \\
& \bar{\phi}(t) \sim-\beta \ln \left(\frac{t}{t_{0}}\right),
\end{aligned}
$$

where $\alpha$ and $\beta$ are constants, and $t_{0}$ is a reference time. Inserting into the dilaton gravity equations gives the following constraints on the constants:

$$
\begin{aligned}
& (D-1) w \alpha+\beta=2 \\
& \beta^{2}+(D-1) \alpha^{2}=2 \beta .
\end{aligned}
$$

Deep in the Hagedorn phase when $w=0$, we get

$$
(\alpha, \beta)=(0,2) .
$$


This corresponds to a static scale factor in the string frame. Converting to the Einstein frame in which the scale factor $\tilde{a}(t)$ is given by

$$
\tilde{a}(t)=a(t) e^{-\phi /(d-1)},
$$

we find

$$
\tilde{a}(t) \sim\left(\frac{t}{t_{0}}\right)^{2 /(d-1)}
$$

In the large $a$ phase when $w=1 / d$, we get

$$
(\alpha, \beta)=\left(\frac{2}{D}, \frac{2}{D}(D-1)\right) .
$$

In this case, the dilaton is constant and hence the string frame and Einstein frame scale factors are the same. As expected, the scale factor evolves as in a standard radiation dominated universe. There is a second solution of (20), but that solution is consistent only for $p=0$.

When $w=-1 / d$, we have

$$
(\alpha, \beta)=\left(-\frac{2}{D}, \frac{2}{D}(D-1)\right) .
$$

The string frame scale factor is expanding as we go backwards in time. Translating to the Einstein frame, we get

$$
\tilde{a}(t) \sim\left(\frac{t}{t_{0}}\right)^{2 /(d-1)} .
$$

In the Einstein frame, the scale factor vanishes at $t=0$, while in the string frame it blows up in this limit.

Let us track the dynamics backwards in time, beginning with a large torus $(R \gg 1)$. The energy will hence be in the momentum modes and the equation of state is that of radiation. As we go back in time, the scale factor decreases (it is the same in the two frames), the energy density increases, and eventually the temperature approaches the Hagedorn value at which point oscillatory and winding modes of the string gas get excited, leading to a transition to an equation of state with $p=0$. We enter a Hagedorn phase during which the string frame scale factor is constant, while the Einstein frame scale factor is decreasing. This means that the radius of the torus $R$ is decreasing, and it soon becomes energetically preferable for the energy of the string gas to drift to the winding modes, leading to an equation of state $w=-1 / d$. In the winding phase, the Einstein frame scale factor is still decreasing, which is a self-consistency check on the assumption that the energy of the string gas is mostly in the winding modes. ${ }^{3}$

\footnotetext{
${ }^{3}$ If we do not allow momentum and winding modes to decay, then, as studied in [21], we obtain solutions where the string frame scale factor oscillates about $a_{0}$.
}
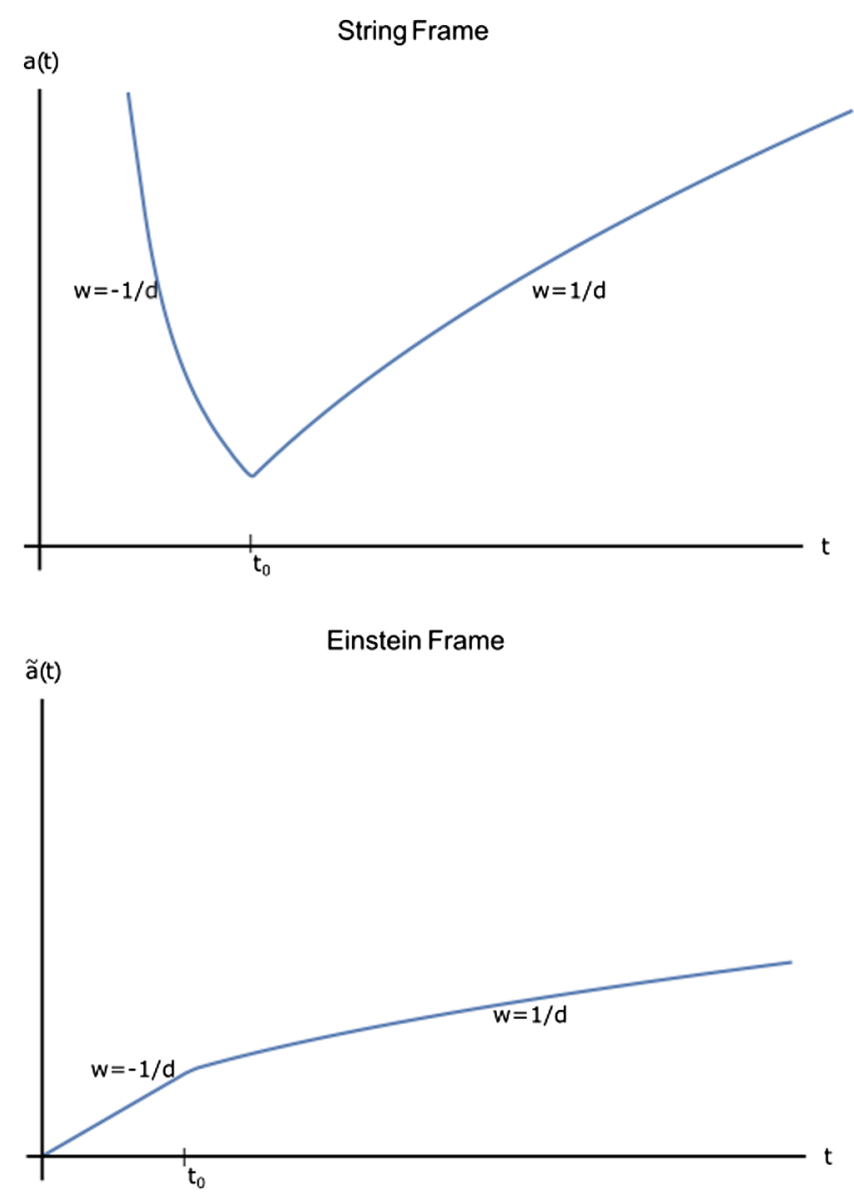

FIG. 2. The schematic solution for the scale factor in the string and Einstein frames for $D=4$. Note that the transition between the winding and momenta equation of state has been smoothed out, as is expected if (15) is considered.

We see that in the string frame, there is no curvature singularity. As the coordinate time $t$ runs from $t=0$ to $t=\infty$, the scale factor is initially contracting, bounces in the Hagedorn phase, and expands afterwards in the radiation phase, as shown schematically in Fig. 2 .

Following [22], we argue that in the phase dominated by winding modes we should measure time in terms of the dual time variable

$$
t_{d} \equiv-\frac{t_{c}^{2}}{t}
$$

where $t_{c}$ corresponds to the coordinate time at the center of the Hagedorn phase. In terms of $t_{d}$, the solution looks like a contracting universe.

From the point of view of the Einstein frame, the scale factor vanishes at $t=0$. But from the point of view of a detector made up of winding modes, the measured scale factor is proportional to $a(t)^{-1}$. Hence, the time interval $0<t<t_{c}$ corresponds to a contracting universe in terms of the dual position basis.

Heuristically, there are two simple reasons for introducing a dual time coordinate. Let us consider for simplicity a 
fixed dilaton, so that we have a radiation solution. It is clear that there is an asymmetry between a large and small scale factor, since the proper time for the scale factor to go to infinity diverges, while it is finite when the scale factor decreases to zero from some finite value. However, from the point of view of T-duality we should not be able to distinguish between a large and a small universe. This is the first hint towards a more general definition of the physical clock, $t_{p}$.

Another qualitative argument follows from special relativity considerations brought together with T-duality. For a large radius, rods are made out of momentum modes, and time measurements for a given physical length, $\Delta x$, are given by

$$
|\Delta t|=|\Delta x|,
$$

where the speed of light has been set to unit. If the universe is composed of closed strings, in principle we could have considered measuring physical length in terms of winding modes as well, and the natural rods built out of these modes are related to the physical length by

$$
\Delta \tilde{x} \rightarrow \frac{\alpha^{2}}{\Delta x}
$$

where $\alpha^{\prime}$ is the string tension. Thus, we can rewrite (28) as

$$
|\Delta \tilde{x}| \rightarrow\left|\frac{\alpha^{\prime 2}}{\Delta t}\right| .
$$

Now, if we cannot distinguish large from small, we could have started the argument using winding modes instead, so that we would write the following relation ${ }^{4}$ :

$$
|\Delta \tilde{x}|=|\Delta \tilde{t}| .
$$

Thus, it is also natural to propose a winding clock that is dual to the momentum clock by combining the above formulas,

$$
|\Delta \tilde{t}| \rightarrow\left|\frac{\alpha^{\prime 2}}{\Delta t}\right|
$$

Evidently, physically speaking there is only a single clock. When only winding or momentum modes are light, the existence of a unique time coordinate is already clear. Around the self-dual point, when both modes are energetically favorable, that should also be the case. Therefore, we need a prescription to reduce both time coordinates to a single physical time. We call this prescription the "physical clock constraint" and it is given by the identification (27).

\footnotetext{
${ }^{4}$ By T-duality one can argue that the dual speed of light is also equal to unit.
}

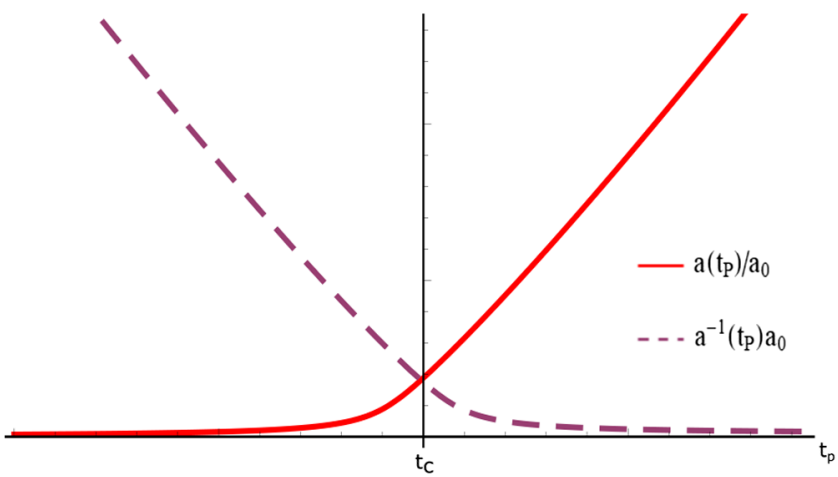

FIG. 3. The scale factor goes to zero only at $t_{p} \rightarrow-\infty$. Similarly its inverse goes to zero when $t_{p} \rightarrow \infty$.

These ideas likely have a very natural interpretation in terms of double field theory [23] (see also [24] for some early work). Double field theory is a generalization of supergravity which lives in $2 d$ spatial dimensions, with the first $d$ dimensions corresponding to the usual $x$ variables, and the second $d$ dimensions to the dual spatial variables $\tilde{x}$. In double field theory there is a generalized metric which, for homogeneous and isotropic cosmology and in the absence of an antisymmetric tensor field, is given by

$$
d s^{2}=-d t^{2}+a^{2}(t) \delta_{i j} d x^{i} d x^{j}+a^{-2}(t) \delta^{i j} d \tilde{x}_{i} d \tilde{x}_{j} .
$$

The determinant of the generalized metric is 1 . As space shrinks in the $x$ directions, it opens up in the $\tilde{x}$ directions. This is sketched in Fig. 3. In a work in progress [25], we are exploring this connection in more detail, in particular using the $O(D, D)$ formalism to formalize the introduction of a dual time and discussing how the physical clock constraint can be seen analogously to the imposition of the section condition in DFT for the dual coordinates [26].

\section{DISCUSSION}

We have studied the equations of motion of a cosmological background containing the scale factor $a(t)$ and the dilaton in the presence of string gas matter sources. Both the background action and the matter action are consistent with the T-duality symmetry of string theory. While we do not expect our description to be adequate in the high density phase when truly stringy effects must be considered, our analysis is an improvement over the usual effective field theory of string cosmology where the underlying background geometry is not covariant with the T-duality symmetry.

We find that the solutions are nonsingular, at least when interpreted in the context of double space-time. We conjecture that an improved description could be obtained using the tools of double field theory. ${ }^{6}$

\footnotetext{
${ }^{5}$ See [27] for a study of cosmological vacuum solutions of double field theory including a dilaton potential.

${ }^{6}$ For a recent paper exploring the required formalism, see [28].
} 


\section{ACKNOWLEDGMENTS}

This research is supported by the IRC-South AfricaCanada Research Chairs Mobility Initiative Grant No. 109684. The research at McGill is also supported in part by funds from Natural Sciences and Engineering Research Council (NSERC) and from the Canada Research Chair program. Two of us (R. B. and G. F.) wish to thank the Banff International Research Station for hosting a very stimulating workshop "String and M-theory Geometries: Double Field Theory, Exceptional Field Theory and Their Applications" during which some of the ideas presented here were developed. G. F. acknowledges financial support from $\mathrm{CNPq}$ (Science Without Borders) and Programme de Bourses d'Excellence pour Étudiants Étrangers (PBEEE)/Quebec Merit Scholarship. G. F. also wishes to thank the University of Cape Town where most of this work was developed. R. C. acknowledges financial support by the South Africa Research Chair National Research Foundation (SARChI NRF) grant holder. A. W. gratefully acknowledges financial support from the Department of Science and Technology and South African Research Chairs Initiative of the National Research Foundation (NRF).
[1] S. W. Hawking and R. Penrose, The singularities of gravitational collapse and cosmology, Proc. R. Soc. A 314, 529 (1970).

[2] A. Borde and A. Vilenkin, Eternal Inflation and the Initial Singularity, Phys. Rev. Lett. 72, 3305 (1994).

[3] A. Borde, A. H. Guth, and A. Vilenkin, Inflationary SpaceTimes Are Incomplete in Past Directions, Phys. Rev. Lett. 90, 151301 (2003).

[4] D. Yoshida and J. Quintin, Maximal extensions and singularities in inflationary spacetimes, Classical Quantum Gravity 35, 155019 (2018).

[5] J. Polchinski, String Theory. Vol. 1: An Introduction to the Bosonic String, Cambridge Monographs on Mathematical Physics (Cambridge University Press, Cambridge, 2007).

[6] J. Polchinski, String Theory. Vol. 2: Superstring Theory and Beyond, Cambridge Monographs on Mathematical Physics (Cambridge University Press, Cambridge, 2007).

[7] R. H. Brandenberger and C. Vafa, Superstrings in the early Universe, Nucl. Phys. B316, 391 (1989).

[8] J. Kripfganz and H. Perlt, Cosmological impact of winding strings, Classical Quantum Gravity 5, 453 (1988).

[9] R. Hagedorn, Statistical thermodynamics of strong interactions at high-energies, Nuovo Cim. Suppl. 3, 147 (1965).

[10] S. P. Patil and R. Brandenberger, Radion stabilization by stringy effects in general relativity, Phys. Rev. D 71, 103522 (2005).S. P. Patil and R. H. Brandenberger, The cosmology of massless string modes, J. Cosmol. Astropart. Phys. 01 (2006) 005; S. Watson and R. Brandenberger, Stabilization of extra dimensions at tree level, J. Cosmol. Astropart. Phys. 11 (2003) 008; S. Watson, Moduli stabilization with the string Higgs effect, Phys. Rev. D 70, 066005 (2004); R. Brandenberger, Y.-K. E. Cheung, and S. Watson, Moduli stabilization with string gases and fluxes, J. High Energy Phys. 05 (2006) 025.

[11] R. H. Brandenberger, String gas cosmology: Progress and problems, Classical Quantum Gravity 28, 204005 (2011); R. H. Brandenberger, String Gas Cosmology, edited by J. Erdmenger (Wiley, New York, 2009), pp. 193-230; T. Battefeld and S. Watson, String gas cosmology, Rev. Mod. Phys. 78, 435 (2006).
[12] N. Deo, S. Jain, and C. I. Tan, String distributions above the Hagedorn energy density, Phys. Rev. D 40, 2626 (1989).

[13] A. Nayeri, R. H. Brandenberger, and C. Vafa, Producing a Scale-Invariant Spectrum of Perturbations in a Hagedorn Phase of String Cosmology, Phys. Rev. Lett. 97, 021302 (2006).

[14] R. H. Brandenberger, A. Nayeri, S. P. Patil, and C. Vafa, String gas cosmology and structure formation, Int. J. Mod. Phys. A 22, 3621 (2007).

[15] V. F. Mukhanov and G. V. Chibisov, Quantum fluctuations and a nonsingular Universe, Pisma Zh. Eksp. Teor. Fiz. 33, 549 (1981) [JETP Lett. 33, 532 (1981)].

[16] P. A. R. Ade et al. (Planck Collaboration), Planck 2015 results. XX. Constraints on inflation, Astron. Astrophys. 594, A20 (2016).

[17] R. H. Brandenberger, A. Nayeri, S. P. Patil, and C. Vafa, Tensor Modes from a Primordial Hagedorn Phase of String Cosmology, Phys. Rev. Lett. 98, 231302 (2007); R. H. Brandenberger, A. Nayeri, and S. P. Patil, Closed string thermodynamics and a blue tensor spectrum, Phys. Rev. D 90, 067301 (2014).

[18] R. Brandenberger, G. Franzmann, and Q. Liang, Running of the spectrum of cosmological perturbations in string gas cosmology, Phys. Rev. D 96, 123513 (2017).

[19] T. Boehm and R. Brandenberger, On T duality in brane gas cosmology, J. Cosmol. Astropart. Phys. 06 (2003) 008.

[20] M. Gasperini and G. Veneziano, Pre-big bang in string cosmology, Astropart. Phys. 1, 317 (1993); The pre-big bang scenario in string cosmology, Phys. Rep. 373, 1 (2003).

[21] A. A. Tseytlin and C. Vafa, Elements of string cosmology, Nucl. Phys. B372, 443 (1992).

[22] R. Brandenberger, R. Costa, G. Franzmann, and A. Weltman, Point particle motion in DFT and a singularityfree cosmological solution, Phys. Rev. D 97, 063530 (2018).

[23] W. Siegel, Superspace duality in low-energy superstrings, Phys. Rev. D 48, 2826 (1993); C. Hull and B. Zwiebach, Double field theory, J. High Energy Phys. 09 (2009) 099. 
[24] M. J. Duff, Duality rotations in string theory, Nucl. Phys. B335, 610 (1990); A. A. Tseytlin, Duality Symmetric String Theory and the Cosmological Constant Problem, Phys. Rev. Lett. 66, 545 (1991); T. Kugo and B. Zwiebach, Target space duality as a symmetry of string field theory, Prog. Theor. Phys. 87, 801 (1992).

[25] R. Brandenberger, R. Costa, G. Franzmann, and A. Weltman (to be published).
[26] G. Aldazabal, D. Marques, and C. Nunez, Double field theory: A pedagogical review, Classical Quantum Gravity 30, 163001 (2013).

[27] H. Wu and H. Yang, Double field theory inspired cosmology, J. Cosmol. Astropart. Phys. 07 (2014) 024.

[28] S. Angus, K. Cho, and J. H. Park, Einstein double field equations, Eur. Phys. J. C 78, 500 (2018). 